\title{
Análise do Padráo Respiratório Durante o uso dos Incentivadores Inspiratórios em Indivíduos Sadios
}

\author{
Respiratory Pattern Analysis During Exercises with Incentive Spirometry in Healthy Subjects
}

\author{
Antonio Adolfo Mattos de Castro ${ }^{1}$, Marcelo Fernandes ${ }^{2}$, Maria Ignês \\ Zanetti Feltrim ${ }^{3}$
}

\section{RESUMO}

O incentivador inspiratório é utilizado para reexpansão pulmonar no paciente pós-operatório, porém poucos dados estão disponíveis sobre seus efeitos no padrão respiratório. Objetivo. Estudar o efeito de dois tipos de incentivadores inspiratórios com e sem o uso de estímulo tátil abdominal na geração de volumes e configuração toracoabdominal. Método. Vinte indivíduos sadios realizaram dois exercícios: a recomendada pelo fabricante (convencional - C) e a proposta pelos autores (modificada $-\mathrm{M}$ ), em dois tipos de aparelhos (Coach ${ }^{\circledast}$ e Respiron $^{\circledast}$ ). A técnica modificada diferiu da convencional pelo estímulo tátil abdominal realizado. Resultados. $\mathrm{O}$ volume corrente no coach C e $M$ foi semelhante $(\mathrm{p}=0,994)$, porém maior do que no Respiron $(p<0,001)$. Não houve diferença nos tempos respiratórios no Respiron e Coach (Respiron C vs $M, p=0,8$; Coach C vs $M, p=0,6$ ). A configuraçáo toracoabdominal apresentou maior participação torácica para o VC quando a técnica convencional foi empregada $(60 \pm 15 \%$ no RC e $61 \pm 13 \%$ no CC; $p<0,001)$. A coordenação toracoabdominal apresentou sincronia durante a realização dos exercícios $(\mathrm{p}=0,232)$. Conclusóes. Houve maior VC gerado com o uso dos incentivadores respiratórios à volume (Coach) do que a fluxo (Respiron), independentemente da técnica empregada; quando realizada a técnica modificada houve maior deslocamento abdominal.

Unitermos. Incentivador inspiratório, Indivíduos Sadios, Padrão Respiratório, Configuração Toracoabdominal, Pletismografia Respiratória por Indutância.

Citaçáo. Castro AAM, Fernandes M, Feltrim MIZ. Análise do Padrão Respiratório Durante o uso dos Incentivadores Inspiratórios em Indivíduos Sadios.

\begin{abstract}
The incentive spirometry is used to expand the lungs in the post-operative patients however few data are available regarding its effects on the respiratory pattern. Objective. Assess the effect of two incentive spirometers with and without the abdominal stimulus in the production of volumes and thoracoabdominal configuration. Method. Twenty healthy subjects accomplished two types of exercises: the proposed by the manufacturer (conventional $-\mathrm{C}$ ) and the proposed by the authors (modified - M), in two types of devices (Coach ${ }^{\oplus}$ and Respiron $\left.{ }^{\circledR}\right)$. The modified technique differed from the conventional by the abdominal stimulus given. Results. Tidal volume in the coach $\mathrm{C}$ and $\mathrm{M}$ was similar $(\mathrm{p}=0.994)$, however higher than in the Respiron $(\mathrm{p}<0.001)$. Respiratory times were similar in the Respiron and Coach within each device (Respiron C vs M, p=0.8; Coach C vs M, p=0.6). Thoracoabdominal configuration presented a higher thoracic participation when the conventional technique was applied $(60 \pm 15 \%$ in the RC and $61 \pm 13 \%$ in the CC; $\mathrm{p}<0.001)$. Thoracoabdominal coordination was synchronic during the exercises $(p=0.232)$. Conclusions. There was a high tidal volume generated with the use of volume-dependent devices (Coach) than flow- dependent ones (Respiron); when the modified technique was used a higher abdominal displacement was found.
\end{abstract}

Keywords. Incentive Spirometry, Healthy Subjects, Respiratory Pattern, Thoracoabdominal Configuration, Respiratory Inductive Pletismography.

Citation. Castro AAM, Fernandes M, Feltrim MIZ. Respiratory Pattern Analysis During Exercises with Incentive Spirometry in Healthy Subjects.
Trabalho realizado no instituto do Coraçáo - InCor - HCFMUSP, Sáo Paulo-SP, Brasil.

1.Fisioterapeuta do Serviço de Fisioterapia do Instituto do Coração (InCor) do Hospital das Clínicas da Faculdade de Medicina da Universidade de São Paulo. Professor do Centro Universitário Adventista de São Paulo - UNASP, São Paulo-SP, Brasil. Professor da Universidade Federal do Pampa - Unipampa.

2.Fisioterapeuta do Serviço de Fisioterapia do Instituto do Coração (InCor) do Hospital das Clínicas da Faculdade de Medicina da Universidade de São Paulo, São Paulo-SP, Brasil.

3.Fisioterapeuta, Doutora, Diretora do Serviço de Fisioterapia do Instituto do Coraçấo (InCor) do Hospital das Clínicas da Faculdade de Medicina da Universidade de São Paulo, São Paulo-SP, Brasil.
Endereço para correspondência: Maria Ignês Zaneti Feltrim

Departamento de Fisioterapia Instituto do Coração - HCFMUSP Av. Dr. Enéas de Carvalho Aguiar, $44-2^{\circ}$ andar Tel.: (11) 3069-5319 CEP 05403-000, São Paulo-SP, Brasil. E-mail: mi.feltrim@incor.usp.br 


\section{INTRODUÇÃO}

O incentivador inspiratório é um aparelho amplamente utilizado, sendo indicado por fisioterapeutas com o objetivo de aumentar o volume corrente e, assim, contribuir para a reexpansão pulmonar em indivíduos submetidos a procedimentos cirúrgicos de laparotomia exploradora, abdominais altos e torácicos ${ }^{1-8}$.

É um recurso mecânico para incentivar o paciente a realizar esforços inspiratórios máximos ${ }^{9,10}$. O incentivo à inspiração resulta em aumento da capacidade inspiratória (CI), em particular, do volume de reserva inspiratório (VRI), o que beneficia, sobretudo, indivíduos no pós-operatório, que adotam padrão respiratório superficial, rápido e monótono ${ }^{11}$.

O padrão respiratório é descrito como a frequência, duração e amplitude da respiraçáo gerando um padrão uniforme e constante. A avaliação do padrão respiratório consiste em monitorar a frequência, volume corrente e os tempos respiratórios gerados ${ }^{12,13}$.

Existem evidências que sugerem que um padrão respiratório superficial, sem inspirações profundas, é o principal fator para alteraçôes da hematose, na mecânica muscular respiratória e nos volumes e capacidades pulmonares. Esses autores mostram as controvérsias existentes sobre a eficácia do incentivador inspiratório na profilaxia de complicações pulmonares pós-operatórias ${ }^{14}$.

Atualmente, no mercado, estão disponíveis diversas marcas e modelos de incentivadores inspiratórios que utilizam dois princípios básicos para atingir o limite imposto pelo esforço inspiratório: fluxo e volume. $\mathrm{O}$ incentivador denominado a fluxo caracteriza-se por gerar fluxo inspiratório rápido, produzindo variação abrupta da pressão transpulmonar em um tempo inspiratório mais curto. O do tipo a volume tem como característica fluxo inspiratório lento, com variação gradual da pressão transpulmonar em um tempo inspiratório mais prolongado ${ }^{14,15}$.

Um dos primeiros estudos analisando a geração de volumes e tempos respiratórios durante o uso de incentivadores inspiratórios foi na década de $80^{11,12}$. Os autores estudaram, por meio do sistema de pletismografia respiratória por indutância, 8 mulheres sadias submetidas à colecistectomia, mostrando que esta forma de exercício respiratório aumentava o deslocamento do compartimento abdominal antes da cirurgia, porém no período pós-operatório esse predomínio não ocorria.

A pletismografia respiratória por indutância é um método eficiente para estudar o movimento toracoabdominal por detectar as alteraçóes na área seccional do tórax e abdome e, assim, inferir o deslocamento do volume pulmonar produzido pelas contraçóes musculares. O método é acurado para avaliar variáveis do padrão respiratório e do movimento toracoabdominal durante repouso e exercício $^{16,17}$.

$\mathrm{Na}$ literatura não estão claros os efeitos do exercício respiratório realizado pelo incentivador inspiratório. Uma revisão de literatura ${ }^{4}$ não pode realizar uma metanálise sobre o assunto e apontam que os presentes estudos apresentam resultados com baixo poder de reprodutibilidade, diferenças na definição de complicações pulmonares pós-operatórias, amostras insuficientes de pacientes, ausência de grupo controle e problemas metodológicos no delineamento dos estudos ${ }^{4}$. Assim sendo, os autores sugerem novos estudos para avaliar a influência da supervisão terapêutica, adesão ao tratamento, posição corporal e custo-efetividade da terapia ${ }^{4,14}$.

Outra técnica bastante difundida entre fisioterapeutas é o estímulo tátil abdominal que consiste em estimular o abdome com as máos no intuito de aumentar a ativação da contração muscular diafragmática e consequentemente orientar um maior volume de ar para as regiōes basais dos pulmóes ${ }^{15}$.

Devido à escassez de dados sobre o assunto, propusemo-nos a estudar o comportamento do movimento toracoabdominal e a geração de volumes pulmonares quando da utilização de incentivador inspiratório, comparando dois tipos de aparelhos - fluxo e volume - e dois tipos de técnicas empregadas com e sem estímulo tátil abdominal, em um grupo de indivíduos sadios.

Hipotetizamos que o volume corrente gerado será maior com o uso de incentivadores do tipo volumétrico (Coach) do que fluxométricos (Respiron) e que o estímulo tátil abdominal estimulará maior participação diafragmática do movimento toracoabdominal. Estes dados são importantes para caracterizar e monitorar as modificaçóes de volumes e tempos respiratórios com o uso dos aparelhos assim como, determinar o componente da parede torácica que ativa-se mais durante a estratégia ventilatória adotada em cada técnica. 


\section{MÉTODO}

\section{Casuística}

Foram estudados 20 indivíduos hígidos, fisioterapeutas, que não referiam história prévia de tabagismo ou doença pulmonar, com valores espirométricos dentro da normalidade. Apesar dos indivíduos estudados terem sido fisioterapeutas, estes não foram informados a respeito do delineamento e hipótese do estudo para evitar que houvesse tendenciosidade nos resultados. A escolha de fisioterapeutas para constituir a amostra ocorreu devido a disponibilidade destes profissionais em nosso hospital.

Critérios de inclusão: Indivíduos hígidos com faixa etária de 20 a 35 anos e que não utilizassem medicaçôes que pudessem alterar os valores medidos (e.g. broncodilatadores).

Critérios de exclusão: Indivíduos que não conseguiram realizar as manobras espirométricas e que não assinaram o termo de consentimento livre e esclarecido.

Após o consentimento informado dos indivíduos e a aprovação da pesquisa pelo Comitê de Ética do Instituto do Coração (InCor) da Faculdade de Medicina da Universidade de São Paulo (SDC 31 17/08/033),

\section{Procedimento}

Realizou-se coleta dos dados antropométricos com medidas de peso e altura para o cálculo do índice de massa corpórea (IMC). A função pulmonar foi avaliada por meio de um espirômetro da marca Puritan-Bennett, modelo PB 100, em concordância com as recomendaçóes da ATS $^{18}$. Foram coletados dados da capacidade vital forçada (CVF), volume expirado forçado no primeiro segundo $\left(V F_{1}\right)$ e relação capacidade vital forçada/volume expirado forçado no primeiro segundo $\left(\mathrm{CVF} / \mathrm{VEF}_{1}\right)$ seguindo as normas da ATS ${ }^{19}$. Todas as medidas foram expressas em litros e porcentagem do previsto.

O movimento toracoabdominal e o volume gerado nos exercícios foram avaliados pelo sistema de pletismografia respiratória por indutância da Noninvasive Monitory Systems, cujo aparelho é denominado Respitrace ${ }^{\circledR}$. Este é composto por transdutores com fios isolantes acoplados a duas faixas elásticas, colocadas no tórax e no abdome, respectivamente. Este mecanismo é excitado por oscilaçôes de alta frequência, detectando-se as distorçôes nas faixas provocadas pelo movimento respiratório ${ }^{16}$. As oscilações são convertidas em curvas gráficas correspondendo ao movimento do tórax, abdome e a soma de ambos os compartimentos. Por meio de um sistema computadorizado os registros gráficos sáo convertidos em volume e tempos respiratórios.

Para realizar a calibração do aparelho, determinaram-se os fatores de escala para tórax e abdome. A calibraçấo foi realizada pelo método dos mínimos quadrados que baseia-se no princípio de que a distribuição do volume inspirado entre os compartimentos do tórax e do abdome varia quando o corpo muda de posição. Neste estudo, foi solicitado que o indivíduo permanecesse na posição sentada, respirando um volume constante de 800 $\mathrm{mL}$, por cinco a seis vezes, a partir de um espirômetro devidamente calibrado. Registravam-se as curvas dos deslocamentos do tórax, abdome e soma dos compartimentos torácico e abdominal. A seguir, foi utilizado um software especialmente construído para medir estas curvas, do qual foi obtido os fatores de escala para tórax e abdome, bem como o índice de correlação entre eles. Quando a correlação dos fatores era inferior a -0,80, rejeitávamos os valores e nova tentativa de calibração era realizada.

Após a calibração do aparelho e ajuste a cada indivíduo, iniciou-se o estudo com a randomização dos exercícios e incentivadores. A randomização ocorreu por meio de sorteio simples para a realização do início das técnicas e dispositivos. $\mathrm{O}$ indivíduo era posicionado sentado ereto e realizava exercícios respiratórios com o incentivador a fluxo ou a volume, de acordo com a técnica recomendada pelo fabricante ou a técnica adaptada pelos autores.

Quando se utilizou o incentivador do tipo a fluxo, marca Respiron ${ }^{\circledR}$, denominou-se Respiron Convencional (RC). O exercício respiratório era realizado seguindo a recomendaçáo do fabricante, que consiste em realizar inspiraçóes máximas, elevando as três esferas presentes no compartimento interno do aparelho. Denominou-se Respiron Modificado (RM) quando o indivíduo realizava três respiraçóes tranquilas em seu volume corrente e, a seguir, inspiraçôes máximas com estímulo manual abdominal de um avaliador que posicionava sua mão na região abdominal do indivíduo e incentivava a deslocar esta região durante o exercício.

No Coach Convencional (CC) utilizou-se o incentivador do tipo a volume, marca DHD Coach ${ }^{\circledR}$. O fabri- 
cante recomenda inspiraçáo lenta e profunda, mantendo o controlador de fluxo no meio do seu curso com elevação simultânea do êmbolo do aparelho ao máximo possível, com pausa inspiratória de seis segundos. Denominou-se Coach Modificado (CM) quando o indivíduo realizava o mesmo protocolo modificado descrito acima porém com o incentivador Coach.

Para cada modalidade o exercício respiratório no incentivador inspiratório era repetido por 5 vezes. $\mathrm{O}$ aparelho foi posicionado à frente do indivíduo sentado para a realização dos exercícios. Dois avaliadores participaram da coleta de dados, sendo que um foi responsável pela orientação prévia da técnica aos participantes do estudo assim como pela padronização do estímulo tátil abdominal e o outro pela monitoração e análise dos dados coletados. A orientação verbal para a realização do estímulo inspiratório nos aparelhos foi feito pelo mesmo avaliador e consistia em estimular o indivíduo a realizar um esforço inspiratório máximo, sustentado ou não, a cada respiração. O estímulo tátil abdominal também foi orientado e realizado pelo mesmo avaliador que posicionava sua mão no abdome do voluntário e incentivava o deslocamento desta regiấo a cada respiração. Dentre as quatro avaliaçóes realizadas houve repouso de 10 minutos para evitar o efeito acumulativo dos exercícios ${ }^{20}$.

\section{Variáveis estudadas}

As variáveis estudadas foram (1) volume corrente (VC) em litros, (2) frequência respiratória (f) em respiraçôes por minuto, (3) tempo inspiratório (TI) em segundos, (4) relação tempo inspiratório/ tempo total (TI/ TTOT), (5) porcentagem de participação do tórax no VC (\%Vtx), (6) porcentagem de participação do abdome no VC (\%Vabd) e (7) a razão da amplitude compartimental máxima sobre o volume corrente (MCA/VC).

\section{Análise Estatística}

Os dados estáo apresentados em valores médios e desvio padrão. Por se tratar de dados paramétricos a comparação entre os aparelhos e as diferentes técnicas em relação a aumento de volume e configuração toracoadominal e a comparação entre as técnicas entre os aparelhos em relação a alteração de tempos respiratórios foram realizadas por meio da análise de variância para medidas de repetição ANOVA dentro de um mesmo grupo (RM one way). Para valores com diferença estatística utilizouse o teste para comparaçóes múltiplas de Tukey, com um nível de significância de $\mathrm{p}<0,05$. Tratando-se de um desfecho que envolve variáveis numéricas contínuas [estímulo tátil abdominal aumenta o volume abdominal (em \%) e volume corrente (em litros)], o tamanho da amostra foi calculado mediante a correlação da variação do aumento da participação do volume corrente abdominal e do volume corrente na técnica com estímulo tátil em uma amostra piloto. Para um erro tipo I de $\alpha=0,05$ e tipo II de $\beta=0,80$ na detecção de alteraçóes dos volumes dos compartimentos abdominal e torácico com a técnica de estímulo tátil abdominal, foi calculada uma amostra mínima de 19 indivíduos.

\section{RESULTADOS}

As características dos indivíduos estão resumidas na Tabela 1. Todos eram jovens, eutróficos e com função pulmonar normal.

Tabela 1

Dados antropométricos e espirométricos dos individuos

\begin{tabular}{c|c}
\hline Variáveis & Média \pm desvio padráo \\
\hline Idade (anos) & $26 \pm 4,74$ \\
Massa corporal $(\mathrm{Kg})$ & $67 \pm 13,2$ \\
Estatura (m) & $1,71 \pm 0,1$ \\
$\mathrm{IMC}(\mathrm{Kg} / \mathrm{m} 2)$ & $22,5 \pm 2,4$ \\
$\mathrm{CVF}(\mathrm{L})$ & $4,5 \pm 1$ \\
$\mathrm{VEF}_{1}(\mathrm{~L})$ & $3,9 \pm 0,8$ \\
$\mathrm{VEF}_{1} / \mathrm{CVF}(\mathrm{L})$ & $0,9 \pm 0,1$ \\
\hline
\end{tabular}

IMC = índice de massa corpórea; $\mathrm{CVF}=$ capacidade vital forçada em litros; $\mathrm{VEF}_{1}(\mathrm{~L})=$ volume expiratório forçado no primeiro segundo em litros; $\mathrm{VEF}_{1}$ / $\mathrm{CVF}=$ razáo volume expiratório forçado no primeiro segundo/capacidade vital forçada em litros.

Os valores médios do VC obtidos durante o uso

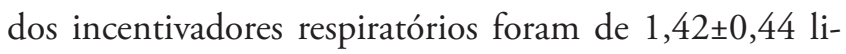
tros no RC, 0,86 $\pm 0,28$ litros no RM, 2,26 $\pm 0,67$ litros no CC e 2,2 $\pm 0,7$ litros no CM. O Coach apresentou maior volume corrente gerado do que o Respiron, independente da técnica empregada $(\mathrm{p}<0,001)$. Entre as técnicas aplicadas o Respiron convencional gerou maior $\mathrm{VC}$ do que o Respiron com a técnica modificada $(\mathrm{p}=0,003)$ (Figura 1).

A participação do abdome no volume corrente 


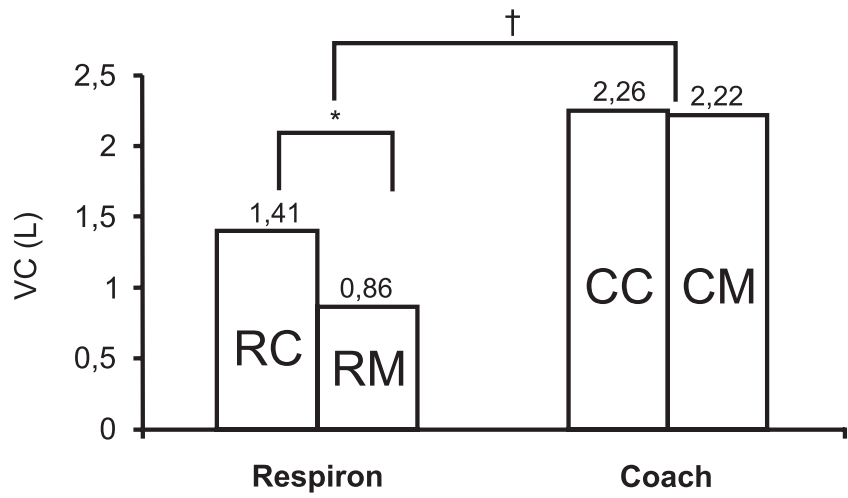

${ }^{*} p<0,05 R C$ versus RM; $\uparrow p<0,05$ Coach versus Respiron

Figura 1. Análise do volume corrente com o uso da técnica convencional (C) e modificada (M) nos incentivadores Respiron (R) e Coach (C).

(Abd\%) apresentou valores médios superiores quando realizou-se a técnica modificada do que com a técnica convencional com $52 \pm 13 \%$ no $\mathrm{RM}$ versus $38 \pm 13 \%$ no $\mathrm{RC}(\mathrm{p}<0,001)$ e $50 \pm 16 \%$ no $\mathrm{CM}$ versus $40 \pm 11 \%$ no CC ( $\mathrm{p}<0,001)$. Por conseguinte, a contribuição do deslocamento torácico em relação ao VC foi maior quando realizou-se a técnica convencional do que com a técnica modificada com $60 \pm 15 \%$ no RC versus $40 \pm 13 \%$ no RM $(\mathrm{p}<0,001)$ e $61 \pm 13 \%$ no $\mathrm{CC}$ versus $50 \pm 15 \%$ no $\mathrm{CM}$ $(\mathrm{p}<0,001)$ (Figura 2).

A coordenação do movimento toracoabdominal medida pelo MCA/VC foi sincrônica com valores médios de $0,98 \pm 0,09$ no RC, $1,01 \pm 0,05$ no $R M, 1,02 \pm 0,07$ no CC e 1,03 $\pm 0,06$ no CM ( $\mathrm{p}=0,232)$.

Os tempos respiratórios foram comparados com a técnica modificada e convencional, porém apenas para cada aparelho, pois estes foram realizados com tempos inspiratórios diferentes orientados pela recomendação do fabricante. $\mathrm{Na}$ realização dos exercícios com o aparelho Respiron a frequência respiratória foi de $13 \pm 4 \mathrm{rpm}$ com a técnica convencional e de $14 \pm 3 \mathrm{rpm}$ na técnica modificada $(\mathrm{p}=0,693)$. As médias dos tempos inspiratórios foram de $1,85 \pm 0,72$ seg. na técnica convencional e 1,78 $\pm 0,49$ seg. na modificada $(\mathrm{p}=0,764)$ e o tempo efetivo da respiraçáo (TI/TTOT) foi de $0,38 \pm 0,11 \mathrm{e}$ $0,39 \pm 0,05$, respectivamente $(\mathrm{p}=0,8)$.
No aparelho Coach, os tempos respiratórios analisados náo apresentaram diferença significante entre as técnicas utilizadas. Os valores médios da frequência respiratória foram de $3 \mathrm{rpm}(\mathrm{p}=0,737)$, tempo inspiratório (TI) de 7,20 $\pm 2,0$ seg. na técnica convencional e $6,80 \pm 2,20 \mathrm{seg}$. na técnica modificada $(\mathrm{p}=0,560)$, tem-

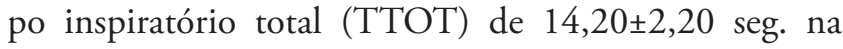
técnica convencional e $14,20 \pm 2,30$ seg. na modificada $(\mathrm{p}=0,925)$, tempo efetivo da respiração (TI/ TTOT) de $0,76 \pm 0,1$ na técnica convencional e $0,75 \pm 0,1$ na modificada $(p=0,9)$ e tempo de pausa de $7,0 \pm 1,20$ seg. na técnica convencional $(\mathrm{p}=0,199)$ e $7,30 \pm 0,70 \mathrm{seg}$. na técnica modificada $(\mathrm{p}=0,199)$ (Tabela 2$)$.

\section{DISCUSSÃO}

Em nosso estudo encontramos os seguintes achados: (1) o aparelho Coach gerou maior volume corrente do que o Respiron, independente da técnica utilizada; (2) ao modificar a técnica dando estímulo tátil na região abdominal e intercalando os exercícios com respirações tranquilas a movimentação abdominal foi maior independente do aparelho utilizado; (3) o movimento toracoabdominal manteve-se sincrônico durante a aplicação do protocolo.

O uso do incentivador inspiratório tem sido estudado para avaliar a sua eficácia no tratamento de complicaçôes pulmonares em indivíduos submetidos aos mais

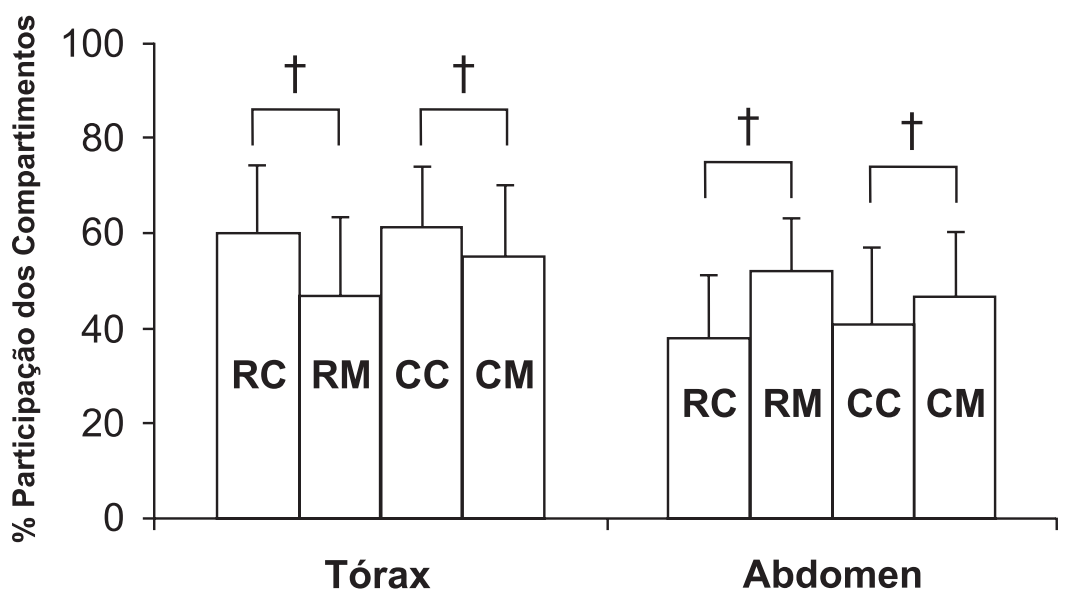

$+\mathrm{p}<0,05$

Figura 2. Análise da participação do deslocamento dos compartimentos torácico e abdominal no volume corrente com o uso da técnica convencional (C) e modificada (M) nos incentivadores Respiron (RC e RM) e Coach (CC e CM). 
Análise da frequência respiratória, tempo inspiratório, tempo de pausa e tempo efetivo da inspiração no incentivador Respiron e Coach

\begin{tabular}{c|c|c|c|c|c|c}
\hline APARELHOS & \multicolumn{3}{|c|}{ RESPIRON } & \multicolumn{3}{c}{ COACH } \\
\hline Modalidade & Convencional & Modificada & $\mathrm{p}$ & Convencional & Modificada & $\mathrm{p}$ \\
\hline $\mathrm{f}(\mathrm{rpm})$ & $13 \pm 4$ & $13 \pm 3$ & 0,7 & $3 \pm 1$ & $3 \pm 1$ & 0,7 \\
\hline $\mathrm{TI}$ (seg.) & $1,85 \pm 0,72$ & $1,78 \pm 0,49$ & 0,8 & $7,2 \pm 2,0$ & $7,38 \pm 2,20$ & 0,6 \\
\hline $\mathrm{T}$ (seg.) & -- & -- & -- & $7,0 \pm 1,20$ & $7,3 \pm 0,7$ & 0,2 \\
\hline TI/TTOT & $0,38 \pm 0,11$ & $0,39 \pm 0,05$ & 0,8 & $0,76 \pm 0,1$ & $0,75 \pm 0,1$ & 0,9 \\
\hline
\end{tabular}

Dados apresentados como média \pm desvio padrão.

$\mathrm{f}(\mathrm{rpm})=$ frequência respiratória em respiraçóes por minuto; TI (seg.) = tempo inspiratório em segundos; $\mathrm{Tp}(\mathrm{seg}$.$) = tempo de pausa em segundos;$ TI/TTOT = razão tempo inspiratório/ tempo total do ciclo respiratório ou tempo efetivo da inspiração.

diversos tipos de procedimentos cirúrgicos $5,6,20,21,22$. No entanto, poucos estudos foram encontrados avaliando o mecanismo de diferentes técnicas em indivíduos sadios. Dois estudos que avaliaram o padrão respiratório em 8 mulheres mostraram que o incentivador inspiratório aumenta o volume corrente nestes indivíduos submetidos à colicistectomia ${ }^{11,12}$. Os autores encontraram que esse aumento ocorria por predomínio do deslocamento abdominal no período pré-operatório; no entanto, no período pós-operatório a manutenção do volume corrente foi devida, primordialmente, pela maior mobilização do compartimento torácico, atribuindo esse resultado à atenção dada pelo paciente no movimento da esfera do inspirômetro do que pela menor ativação neural diafragmática ${ }^{11}$.

Outro estudo ${ }^{13}$ avaliou, por meio do sistema de pletismografia respiratória por indutância, o volume corrente e o movimento toracoabdominal em 7 indivíduos sadios, realizando exercícios com incentivadores inspiratórios do tipo volume dependente (Coach e Voldyne) e fluxo dependente (Triflo II e Respirex). Os exercícios com incentivadores a volume contribuíram para gerar maior volume com menor frequência respiratória. Esses resultados também foram observados em nosso estudo quando aplicamos o aparelho Coach, independente da técnica. Neste aparelho a inspiração é realizada por um tempo prolongado, o que propicia maior ativação dos músculos inspiratórios ${ }^{11}$, com aumento da pressão transpulmonar e do volume corrente.

Em um outro estudo que comparou o efeito de um incentivador a volume e de fluxo mostrou que indivíduos sadios ao utilizar o incentivador Triflo II (fluxo) aumentaram suas frequências respiratórias e ativação eletromio- gráfica do músculo esternocleidomastóideo, assim como diminuíram os seus tempos inspiratórios (TI ${ }^{21}$.

Nós encontramos que no aparelho Respiron o TI é menor. As contrações musculares são rápidas (menor TI/ TTOT), com menor geração de força muscular e menor deslocamento do ar inspirado (menor VC).

Nós propusemos modificar a técnica recomendada pelo fabricante incluindo o estímulo manual na região abdominal e 3 respiraçôes tranquilas entre os esforços inspiratórios máximos. Observamos que no Respiron modificado houve maior movimento do compartimento abdominal expressando maior deslocamento diafragmático, porém o volume corrente mobilizado foi menor quando comparado aos esforços inspiratórios realizados em sequência. Esta foi a técnica na qual o tórax contribuiu menos para a geração do volume corrente. Provavelmente, ao executar respiraçóes diafragmáticas com tempo inspiratório curto, houve menor variação da pressão intratorácica, o que explica menor geração de volume corrente.

O tórax foi o compartimento que mais se mobilizou durante as técnicas empregadas, exceto a do Respiron modificada. Este fato vem reforçar que esses aparelhos contribuem para o aumento do volume corrente provavelmente em função da maior ativação dos músculos da caixa torácica. Sua indicação, portanto, parece ser mais recomendada para os casos de diminuição de volumes pulmonares nas regiōes superiores e médias. Para maior ativação do músculo diafragma deve-se usar aparelhos a volume; quando o incentivador a fluxo é empregado recomendamos intercalar os exercícios com respiraçóes tranquilas e estímulo tátil abdominal.

Do mesmo modo foram avaliados diferentes in- 
centivadores inspiratórios em relaçáo ao volume, tempos e configuração toracoabdominal de indivíduos sadios ${ }^{13}$. Assim como os nossos dados, os autores mostraram que houve aumento da participaçáo abdominal com a utilização dos incentivadores a volume. Também mostraram que os incentivadores a fluxo geraram maior contração muscular (TI/TTOT) intercostal (maior movimentação de caixa torácica) do que outros aparelhos ${ }^{20}$.

A coordenação entre os compartimentos torácico e abdominal (MCA/VC) não apresentou diferença estatística significante entre os aparelhos e as técnicas empregadas. Podemos, então, supor que, quando estes indivíduos realizaram os exercícios com qualquer aparelho não houve incoordenação de movimento toracoabdominal, o que mostra uma adequada estratégia ventilatória durante o esforço. Nossos dados se assemelham a de outros estudos que avaliaram à sincronia de movimento toracoabdominal durante o uso de incentivadores inspiratórios em indivíduos sadios.

Há evidências na literatura quanto a análise do comportamento toracoabdominal de indivíduos sadios com o auxílio de equipamentos com o eletromiógrafo e pletismógrafo. Estes estudos mostraram que durante o uso de incentivadores inspiratórios não há alteração desproporcional da contração muscular ou da configuração toracoabdominal da parede torácica ${ }^{20,21}$.

Quando analisamos os tempos respiratórios no aparelho Coach, eles foram longos devido a pausa de 6 segundos no final da inspiração, recomendada pelo fabricante e seguida neste estudo. Esta é a razão do baixo fluxo inspiratório e aumento gradual da pressão transpulmonar e do volume corrente.

Outros autores também mostraram que o uso dos incentivadores a volume em indivíduos sadios, independente do uso da pausa de 6 segundos, aumentou o tempo inspiratório e gerou um fluxo inspiratório lento e gradual até o máximo de volume corrente inspirado atingido, mecanismo esse, diferente do incentivador a fluxo ${ }^{21}$.

Este estudo possui a limitação do fato de não termos analisado os volumes e capacidades pulmonares não mobilizáveis (e.g., volume residual, capacidade residual funcional, capacidade pulmonar total) do pulmão o que poderia ter influenciado o padrão respiratório e configuração toracoabdominal dos indivíduos pesquisados. No entanto, minimizamos esse fator confundidor devido ao fato dos indivíduos terem sido controles deles mesmos.

Por fim, este estudo possui uma relevante aplicabilidade prática, pois mostra que para atingir adequada expansão pulmonar a técnica a ser empregada deve ser a do coach convencional, pois este incentivador é o que gera maior volume corrente. A técnica segundo as recomendaçôes do fabricante é facilmente realizada pelo indivíduo sem a necessidade de muitas intervençóes do fisioterapeuta, assim, tornando a terapia custo-efetiva.

\section{CONCLUSÕES}

Concluímos que em indivíduos sadios os aparelhos Respiron e Coach geram maior volume corrente do que o período de repouso, porém o aparelho Coach gerou maior volume corrente do que o Respiron, independente da técnica utilizada; (2) a movimentação da caixa torácica é maior na execução dos exercícios com o uso da técnica convencional, no entanto, ao modificar a técnica dando estímulo tátil na região abdominal e intercalando os exercícios com respirações tranquilas a movimentação abdominal foi maior independente do aparelho utilizado; (3) o movimento toracoabdominal manteve-se sincrônico durante a aplicação do protocolo.

\section{REFERÊNCIAS}

1.Celli BR, Rodriguez KS, Snider GL. A controlled trial of intermittent positive pressure breathing, incentive spirometry, and deep breathing exercises. Am Rev Respir Dis 1984;130:12-5.

2.Dull JL, Dull WL. Are maximal inspiratory breathing exercises or incentive spirometry better than early mobilization after cardiopulmonary bypass? Phys Ther 1983;63:655-9.

3.Krastins IRB, Corey ML, McLeod A, Edmonds J, Levinson H, Moes F. An evaluation of incentive spirometry in the management of pulmonary complications after cardiac surgery in a pediatric population. Crit Care Med 1982;10:525-8.

http://dx.doi.org/10.1097/00003246-198208000-00009

4.Overend, TJ, Anderson CM, Lucy SD, Bhatia C, Jonsson BI, Timmermans C. The effect of incentive spirometry on postoperative pulmonary complications. Chest 2001;120:971-8.

http://dx.doi.org/10.1378/chest.120.3.971

5.Gale GD, Sanders DE. The Bartlett-Edwards Incentive Spirometer: A preliminary assessment of its use in the prevention of atelectasis after cardio-pulmonary bypass. Canad Anaesth Soc J 1977;24:408-16.

http://dx.doi.org/10.1007/BF03005115

6.Gale GD, Sanders DE. Incentive spirometry: Its value after cardiac surgery. 
Canad Anaesth Soc J 1980;27:475-80.

http://dx.doi.org/10.1007/BF03007047

7. Matte P, Jacquet L, Van Dyck M, Goenen M. Effects of conventional physiotherapy, continuous positive airway pressure and non-invasive ventilatory support with bilevel positive airway pressure after coronary artery bypass grafting. Acta Anaesthesiologica Scandinavica 2000;44:75-81.

http://dx.doi.org/10.1034/j.1399-6576.2000.440114.x

8.Schwieger I, Gamulin Z, Foster A, Meyer P, Gemperle M, Suter PM. Absence of benefit of incentive spirometry in low-risk patients undergoing elective cholecystectomy. Chest 1986;89:652-5.

http://dx.doi.org/10.1378/chest.89.5.652

9.Stock MC, Downs JB, Cooper RB, Lebenson IM, Cleveland J, Weaver $\mathrm{DE}$, et al. Comparison of continuous positive airway pressure,incentive spirometry and conservative therapy after cardiac operations. Crit Care Med 1984;12:969-72.

http://dx.doi.org/10.1097/00003246-198411000-00010

10.Stock MC, Downs JB, Guawer PK, Alster JM, Imrey PB. Prevention of postoperative pulmonary complications with CPAP, incentive spirometry, and conservative therapy. Chest 1985;87:151-6.

http://dx.doi.org/10.1378/chest.87.2.151

11.Chuter TAM, Weissman C, Gump FE. Effect of incentive spirometry on diaphragmantic function after surgery. Surgery 1989;105:488-93.

12.Chuter TAM, Weissman C, Mathews DM, Starker P M. Diaphragmatic breathing maneuvers and movement of the diaphragm after cholecystectomy. Chest 1990;97:1110-4.

http://dx.doi.org/10.1378/chest.97.5.1110
13.Parreira VP, Tomich GM, Britto RR, Sampaio RF. Assessment of inspired volume (VI) and thoracoabdominal motion, using volume and flow-oriented incentive spirometers (IS), in healthy subjects. Eur Respir J 2000;20:180-1.

14.Matos JP, Madureira KT, Filho DS, Parreira, VF. Eficácia da espirometria de incentivo na prevenção de complicaçôes pulmonares após cirurgias torácicas e abdominais - revisão de literatura. Rev Bras Fisioter 2003;7:93-9.

15.Feltrim MIZ, Jardim JRB. Movimento toracoabdominal e exercícios respiratórios: revisão de literatura. Rev Fisioter Univ São Paulo 2004;11:105-13. 16.Conh MA, Rao ASV, Broudy M, Birch S, Watson H, Atkins N. The respiratory inductive pletismograph: a new non-invasive monitor. Bull Eur Physiopathol Respir 1982; 18:643-58.

17.Konno K, Mead J. Measurement of the separate volume changes of rib cage and abdome during breathing. J Appl Physiol 1967; 22:407-22.

18.American Thoracic Society (ATS). Standards for the diagnosis and care of patients with chronic obstructive pulmonary disease. Am J Respir Crit Care Med 1995;152:s78-s121.

19.American Thoracic Society. Lung function testing: selection of reference values and interpretative strategies. Am Rev Respir Dis 1995;144:1202-24.

20.Parreira VF, Tomich GM, Britto RR, Sampaio RF. Assessment of tidal volume and thoracoabdominal motion using volume and flow-oriented incentive spirometers in healthy subjects. Braz J Med Biol Res 2005;38:1105-12. http://dx.doi.org/10.1590/S0100-879X2005000700014

21.Tomich GM, França DC, Diório AC, Britto RR, Sampaio RF, Parreira VF. Breathing pattern, thoracoabdominal motion and muscular activity during three breathing exercises. Braz J Med Biol Res 2007;40:1409-17.

http://dx.doi.org/10.1590/S0100-879X2006005000165

22.Pullen RL. Teaching bedside incentive spirometry. Nursing 2003;33:24. 